\title{
ARTICLE
}

\section{Development and characterization of an inducible Dicer conditional knockout mouse model of Parkinson's disease: validation of the antiparkinsonian effects of a sigma-1 receptor agonist and dihydromyricetin}

\author{
Chen-hong Guo ${ }^{1}$, Ting $\mathrm{Cao}^{1}$, Long-tai Zheng ${ }^{1}$, John L Waddington ${ }^{1,2}$ and Xue-chu Zhen ${ }^{1}$
}

Parkinson's disease (PD) is a common neurodegenerative disease characterized by motor impairment and progressive loss of dopamine (DA) neurons. At present, the acute application of neurotoxic drugs such as 1-methyl-4-phenyl-1,2,3,6-tetrahydropyridine (MPTP) and 6-hydroxydopamine (6-OHDA) are commonly used to simulate the pathology of PD; however, it is difficult to induce the progressive pathogenesis of PD with these models. In this study, we employed DAT promoter-mediated Cre transgenic mice to establish tamoxifen-inducible Dicer conditional knockout (CKO) mice in an effort to mimic the progressive loss of DA neurons and the development of PD-like behavioral phenotypes. The results showed that Dicer cKO mice exhibited progressive loss of DA neurons in the substantia nigra (SN) following tamoxifen administration. Significant DA loss was observed 6 weeks after tamoxifen administration; accordingly, progressive motor function impairment was also observed. We also found that a significant neuroinflammatory response, as evidenced by microglial proliferation, another hallmark of PD pathogenesis, accompanied the loss of DA neurons. The acute application of levo-DOPA (L-DOPA) relieved the PD-like motor impairments in Dicer cKO mice to exert its antiparkinsonian action, indicating that the model can be used to evaluate the antiparkinsonian efficacy of PD drugs. To further elucidate the potential application of this novel PD animal model for PD drug development, we employed the powerful neuroprotective agent dihydromyricetin (DHM) $(10 \mathrm{mg} / \mathrm{kg})$ and the selective sigma-1 receptor agonist PRE- $084(1 \mathrm{mg} / \mathrm{kg}), \mathrm{both}$ of which were previously shown to produce antiparkinsonian effects. The results indicated that the chronic administration of either DHM or PRE-084 attenuated the Dicer CKO-induced loss of DA neurons and motor impairments, although the two drugs acted through different mechanisms. These data indicate that the Dicer cKO mouse model may be a useful model for investigating the pathological development of PD and intervention-mediated changes. In conclusion, this transgenic mouse model appears to simulate the progressive pathogenesis of PD and may be a potentially useful model for PD drug discovery.

Key words: Parkinson's disease; Dicer; gene knockout; dihydromyricetin; PRE-084

Acta Pharmacologica Sinica (2020) 41:499-507; https://doi.org/10.1038/s41401-020-0379-5

\section{INTRODUCTION}

Parkinson's disease (PD) is characterized by progressive damage to dopamine (DA) neurons in the midbrain substantia nigra (SN) $[1,2]$. 1-Methyl-4-phenyl-1,2,3,6-tetrahydropyridine (MPTP), 6hydroxydopamine (6-OHDA), and other neurotoxic compounds are commonly used in the laboratory to establish PD models. These neurotoxins are selectively taken up by DA neurons and are mainly targeted to the mitochondrial complex, eventually causing the death of DA neurons, and they have been widely used for PD drug evaluation and pathological studies of diseases [3, 4]. However, these neurotoxin-induced animal models cause acute death of DA neurons and therefore do not simulate the progressive pathogenesis of PD. Thus, there is an unmet need to develop novel animal models that resemble the chronic pathological development of PD.
MicroRNAs (miRNAs) are single-stranded noncoding RNAs composed of 22 nucleotides that play an important role in neuronal development and function in both physiological and disease conditions [5-7]. Dicer is a key enzyme in the progression of miRNA synthesis. Dicer-specific knockout of hippocampal neurons using the calmodulin kinase (Camk II) promoter induces hippocampal neuronal death and neuroinflammation [8]; Dicerspecific knockout in the cerebellum also causes Purkinje cell death and behavioral ataxia in mice [9]. Moreover, Kim and colleagues constructed a mouse line with Dicer conditional knockout (cKO) driven by the DAT promoter using Cre recombinase, revealing the key role of miRNA in DA neuronal survival for the first time. They found that the loss of DA neurons began 3 weeks after Dicer deletion and that 90\% DA cell death was reached after 8 weeks [10]. In addition, Pang et al. employed a different approach, using

\footnotetext{
${ }^{1}$ Jiangsu Key Laboratory of Neuropsychiatric Diseases and College of Pharmaceutical Sciences, Soochow University, Suzhou 215123, China and ${ }^{2}$ School of Pharmacy and Biomolecular Sciences (PBS), Royal College of Surgeons in Ireland, Dublin, Ireland

Correspondence: Xue-chu Zhen (zhenxuechu@suda.edu.cn)
}

Received: 15 December 2019 Accepted: 13 February 2020

Published online: 28 February 2020 
virus-mediated Cre expression in adult floxed Dicer knockin mice and found that the motor ability of mice was impaired due to the loss of DA neurons in the $\mathrm{SN}$, further confirming the importance of Dicer for the survival of DA neurons [11]. These studies revealed that Dicer depletion-induced DA neuronal death is a progressive process that is distinct from the neurotoxin-induced acute death of DA neurons and that may somehow resemble the progressive death in PD pathology. However, the detailed pathological changes related to Dicer depletion and behavioral alterations remain to be addressed. Moreover, the potential value of this model for PD drug evaluation requires further study. In addition, given that $P D$ is an aging-related disease, inducible Dicer depletion in DA neurons in adult animals may be a better approach for mimicking the pathology of PD. For this purpose, we developed DAT promoter-mediated Cre transgenic mice to establish an inducible Dicer cKO mouse line to mimic progressive DA neuron loss. We first characterized developmental changes in both behavior and pathology following Dicer CKO in DA neurons. These results indicated that Dicer CKO in DA neurons in response to tamoxifen administration in adult mice induced the progressive development of PD-like phenotypes. We further investigated the potential significance of the Dicer CKO mouse model for PD drug evaluation by comparing the effects of various compounds, such as levo-DOPA ( $L$-DOPA), the neuroprotective agent dihydromyricetin (DHM) and a sigma-1 receptor agonist, which were previously shown to elicit promising anti-PD effects through different mechanisms. These results clearly indicated that the Dicer cKO mouse model can serve as an alternative experimental PD model for drug development with the advantage of mimicking the progressive development of PD pathology.

\section{MATERIALS AND METHODS}

\section{Inducible Dicer CKO mouse preparation}

Floxed Dicer mice $\left(\right.$ Dicer $\left.^{\mathrm{f} / \mathrm{f}}\right)$ and DAT-icreER transgene mice were donated by Dr. Xinyuan Lv. (Medical College of Georgia, USA) and bred under SPF conditions (temperature: $22 \pm 1{ }^{\circ} \mathrm{C}$, humidity: $55 \%$ $\pm 5 \%, 12$-h light-dark cycle) with free access to food and water. All animal experiments were approved by the Institutional Animal Care and Use Committee of Soochow University and were in accordance with the Guidelines for the Care and Use of Laboratory Animals. Dicer ${ }^{\mathrm{f} / \mathrm{f}}$ mice were crossed with DAT-icreER mice to obtain DAT-icreER;Dicer ${ }^{\mathrm{f} /+}$ mice, the latter were bred with Dicer ${ }^{\mathrm{f} / \mathrm{f}}$ mice to produce DAT-icreER;Dicer ${ }^{\mathrm{f} / \mathrm{f}}$ mice; these mice were regarded as Dicer CKO mice, and their littermates were used as controls. The genotypes of the mice were determined by PCR using genomic DNA from the toes of the mice and the following primer sequences:

(1) Dicer: forward 5'-CCTGACAGTGACGGTCCAAAG-3' reverse 5'-CATGACTCTTCAACTCAAACT-3'.

(2) Cre transgene: forward 5'-AGACTTCCTCGGGCTCCCG-3' reverse $5^{\prime}$-TGGCTTGCAGGTACAGGAGG-3'.

(3) positive control: forward: 5'-CAAATGTTGCTTGTCTGGTG-3' reverse: 5'-GTCAGTCGAGTGCACAGTIT-3'.

The PCR products were used to determine the genotypes of the mice; a 300-bp band indicated the Cre transgene, a 200-bp band indicated the positive control, a 351-bp band indicated WT Dicer, and a 420-bp band indicated Dicer ${ }^{\mathrm{f} / \mathrm{f}}$.

\section{Drug treatment}

Dicer deletion was induced in 8- to 10-week-old mice. Briefly, $1 \mathrm{mg}$ of tamoxifen (Sigma-Aldrich, USA, T5648) was diluted in sunflower oil at a final concentration of $10 \mathrm{mg} / \mathrm{mL}$ and injected intraperitoneally (i.p.) twice a day over five consecutive days. The animals then received different treatments: (1) tamoxifen administration alone: the mice were used to observe pathological and behavioral changes at various times following Dicer CKO; (2) the L-DOPA treatment group: the mice were injected with $8 \mathrm{mg} / \mathrm{kg} \mathrm{L-DOPA} \mathrm{(Sigma-Aldrich,} \mathrm{USA,} \mathrm{D9628)} \mathrm{combined}$ with $15 \mathrm{mg} / \mathrm{kg}$ benserazide (Sigma-Aldrich, USA, B7283) 8 weeks after tamoxifen administration to validate the antiparkinsonian effect of L-DOPA through behavioral measurements; (3) DHM treatment: the mice were administered tamoxifen followed by daily injection of $10 \mathrm{mg} / \mathrm{kg}$ DHM (Nature Standard, China, ST03840120MG) for 8 weeks to evaluate the neuroprotection and antiparkinsonian effects of the drug; and (4) Sigma-1 receptor agonist treatment: PRE-084 (MCE, USA, HY-18100A) was injected daily together with tamoxifen for consecutive 8 weeks, and the antiparkinsonian effects were evaluated weekly after 6 weeks of tamoxifen administration.

\section{Behavioral experiments}

Pole test and rotarod test: The experiments were performed as previously reported by our group $[12,13]$. In brief, before the experiments, the mice were trained to crawl downward. During the experiment, the mice were placed head down at the top of a rough wooden pole $(60 \mathrm{~cm}$ length, $2 \mathrm{~cm}$ in diameter), and the time required for the mice to descend from the top of the pole to the ground was recorded. The experiment was repeated three times for each mouse. For the rotarod test, the mice were first trained until they were able to remain on the rotarod for more than $120 \mathrm{~s}$ at a speed of 20 revolutions per minute $(r / m i n)$. During the experiment, the speed of the rotarod was increased from 20 to $40 \mathrm{r} / \mathrm{min}$ within $5 \mathrm{~min}$. The mice were allowed to walk freely on the rotarod, and the latency time to fall from the rotarod was recorded. The measurement was repeated three times for each mouse.

Western blotting

After the behavioral tests, the mice were sacrificed to collect brain tissues for biological tests. Total protein isolated from mouse SN tissues were lysed in RIPA buffer and denatured at $95^{\circ} \mathrm{C}$ for $5 \mathrm{~min}$. Protein concentrations were determined using a BCA Protein Assay Kit. Proteins $(20 \mu \mathrm{g})$ were loaded on SDS-PAGE gels and transferred to PVDF membranes for $2 \mathrm{~h}$ at $250 \mathrm{~mA}$. The membranes were incubated with $5 \%$ nonfat milk for $2 \mathrm{~h}$ at room temperature before incubation with the respective antibodies, including anti-TH (1:1000; Millipore, USA), anti-a-tubulin (1:10,000; Sigma-Aldrich, USA), and anti- $\beta$-tubulin $(1: 10,000$; Sigma-Aldrich, USA), at $4{ }^{\circ} \mathrm{C}$ overnight. The membranes were washed three times with TBST. The membranes were incubated with respective mouse IgG (1:10,000; Sigma-Aldrich, USA) or rabbit IgG (1:10,000; Sigma-Aldrich, USA) secondary antibodies for $2 \mathrm{~h}$ at room temperature. The results were analyzed using ImageJ software.

Immunofluorescence staining

After the behavioral tests, some mice were anesthetized with $4 \%$ chloral hydrate and then perfused through the left ventricle with PBS ( $\mathrm{pH} 7.4$ ) followed by $4 \%$ paraformaldehyde in PBS. Mouse brains were collected and postfixed in paraformaldehyde overnight at $4{ }^{\circ} \mathrm{C}$ and then dehydrated in $30 \%$ sucrose at $4{ }^{\circ} \mathrm{C}$ for 3 days. The brain tissues were serially cut into $20-\mu \mathrm{m}$ coronal sections using a freezing microtome. For immunofluorescence staining, the sections were washed three times with PBS and incubated in PBS containing 3\% BSA and 0.3\% Triton for $2 \mathrm{~h}$ at room temperature. The sections were then incubated with an antiTH (1:400, Millipore, USA) or anti-lba1 (1:400; Wako, Japan) antibody for $24 \mathrm{~h}$ at $4{ }^{\circ} \mathrm{C}$. Then, the sections were washed in PBST (PBS containing $0.3 \%$ Triton) and incubated with Alexa Fluor 488conjugated goat anti-mouse IgG (1:400; Thermo Fisher Scientific, USA) or Alexa Fluor 594-conjugated goat anti-rabbit IgG (1:400; Thermo Fisher Scientific, USA) for $2 \mathrm{~h}$ at room temperature in the dark. The sections were washed and then mounted using mounting medium. Images were captured using a laser confocal fluorescence microscope. 

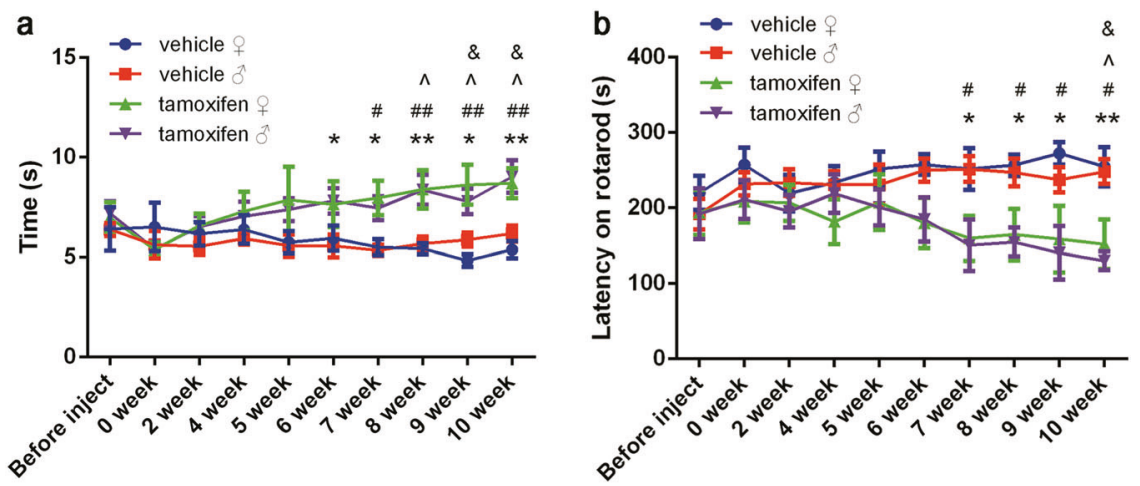

Fig. 1 Dicer cKO mice exhibited a progressive impairment in motor activities. Eight- to 10 -week-old mice were injected with $10 \mathrm{mg} / \mathrm{ml}$ tamoxifen (i.p.) twice a day for 5 days. The basal motor activity was monitored before drug administration and after last drug injection. After last injection, mice were measured by a pole test and $\mathbf{b}$ rotarod test every other week. Data were presented as mean \pm SEM. Statistical analyses were performed by two-way ANOVA. $n=8$ per group. ${ }^{*} P<0.05,{ }^{*} P<0.01$; tamoxifen ${ }^{*}$ vs vehicle ${ }^{*}$. ${ }^{\#} P<0.05$, ${ }^{\# \#} P<0.01$; tamoxifen + vs vehicleo. ${ }^{\wedge} P<0.05$; tamoxifen ${ }^{\wedge}, 0$ week vs time after tamoxifen administration. ${ }^{\&} P<0.05$; tamoxifen $\$$, 0 week vs time after tamoxifen administration)

\section{Statistical analysis}

Data analysis was performed by using GraphPad Prism 6.0 software. The results are expressed as the mean \pm standard error of the mean (SEM). One-way or two-way ANOVA was used for multiple-group comparisons. ${ }^{*} P<0.05$ was considered statistically significant.

\section{RESULTS}

Dicer CKO mice showed a progressive decline in motor ability and damage to DA neurons

We first examined changes in the motor ability of the mice after tamoxifen administration to induce Dicer cKO in DA neurons at different time points. As shown in Fig. 1, impairments in motor activity were observed at week 6 and worsened progressively with time. There was no difference in motor activity between male and female animals. In association with this observation, we also detected the progressive loss of DA neurons in the $\mathrm{SN}$, as measured by tyrosine hydroxylase (TH) expression using Western blotting. This was further confirmed by immunostaining with an anti-TH antibody (Fig. 2). These data confirmed that the induction of Dicer CKO in DA neurons in adult mice resulted in progressive DA neuronal loss and that the mice progressively developed a PDlike phenotype. These results indicated that these transgenic animals may represent a novel animal model for the study of PD, particularly the progressive development of PD-like behavioral and pathological phenotypes, that may be a useful tool for evaluating the protection of DA neurons or for studying the mechanism of the effects of interventions or drugs.

Progressive neuroinflammation during PD-like pathological development in Dicer cKO mice

Neuroinflammation is believed to contribute to the development of PD. The modulation of neuroinflammation has become an important drug target for neurodegenerative diseases, including PD [14-16]. To elucidate the inflammatory response during the development of PD-like phenotypes in the Dicer CKO mice, the animals were sacrificed and their brains were collected 4, 6, and 8 weeks after tamoxifen administration. Immunofluorescence staining for the microglial marker lba1 was performed (Fig. 3). The results revealed a progressive increase in the number of lba1 immunopositive cells from week 6 , which coincided with the onset of DA neuronal death. The above results revealed that Dicer knockout can cause progressive neuroinflammation in the $\mathrm{SN}$, which is one of the hallmarks of pathology in PD.
Acute application of $L$-DOPA to Dicer CKO mice relieved PD-like motor impairments

We characterized the PD-like behavioral and pathological phenotypes in inducible Dicer cKO mice. We next tested the potential application of this novel model to study the efficacy of PD drugs. Dicer cKO mice, which steadily developed PD-like behaviors in response to tamoxifen induction, were administered $L$-DOPA, and the results indicated that $L$-DOPA treatment significantly relieved motor impairments, as evidenced by performance on the pole test and rotarod test (Fig. 4), indicating that the Dicer CKO mouse line can be used as an alternative animal model for evaluating the efficacy of PD drugs.

Chronic application of the neuroprotective agent DHM or the sigma-1 receptor agonist PRE-084 attenuated DA neuronal damage and behavioral abnormalities in Dicer CKO mice To further evaluate the potential application of Dicer cKO mice in PD research, we investigated whether this animal model can be used to study the preventive effect of neuroprotective agents or antiparkinsonian agents on PD pathological development given the progressive nature of PD-like pathology in Dicer CKO mice. To this end, we employed DHM, a powerful neuroprotective agent that was previously shown to protect DA neuron [13], and PRE084, a selective sigma-1 receptor agonist, since the modulation of the sigma-1 receptor was recently identified an important target for PD drug discovery and treatment [17]. Dicer CKO mice were administered DHM, PRE-084, or vehicle for 8 consecutive weeks, and the drug treatments were started after tamoxifen induction. Behavioral tests were performed every other week starting from week 6 following tamoxifen administration. After the behavioral tests, the mice were sacrificed for biological tests (Fig. 5a). The results showed that, in comparison with tamoxifen alone, DHM treatment significantly attenuated Dicer cKO-induced impairments in motor functions (Fig. 5b, c). Similarly, the chronic administration of the selective sigma-1 receptor agonist PRE-084 also dramatically protected against impairments in motor functions (Fig. 5d, e).

In support of these findings, the Western blot results indicated that after DHM administration, the expression of $\mathrm{TH}$ was significantly higher than that in the control (no drug treatment group) (Fig. 6a, b). Meanwhile, we found that chronic DHM treatment significantly reduced the number of microglial cells, suggesting a decrease in neuroinflammation (Fig. 6c-e). In addition, treatment with the sigma-1 receptor agonist PRE-084 also significantly restored $\mathrm{TH}$ expression (Fig. 7a, b) and 
a

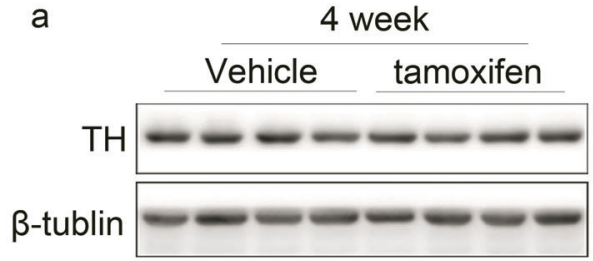

8 week

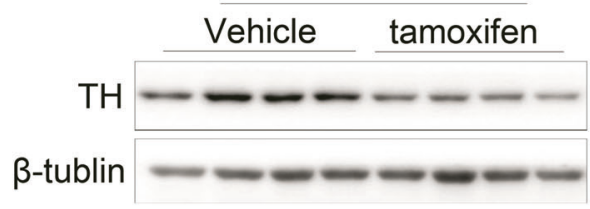

b
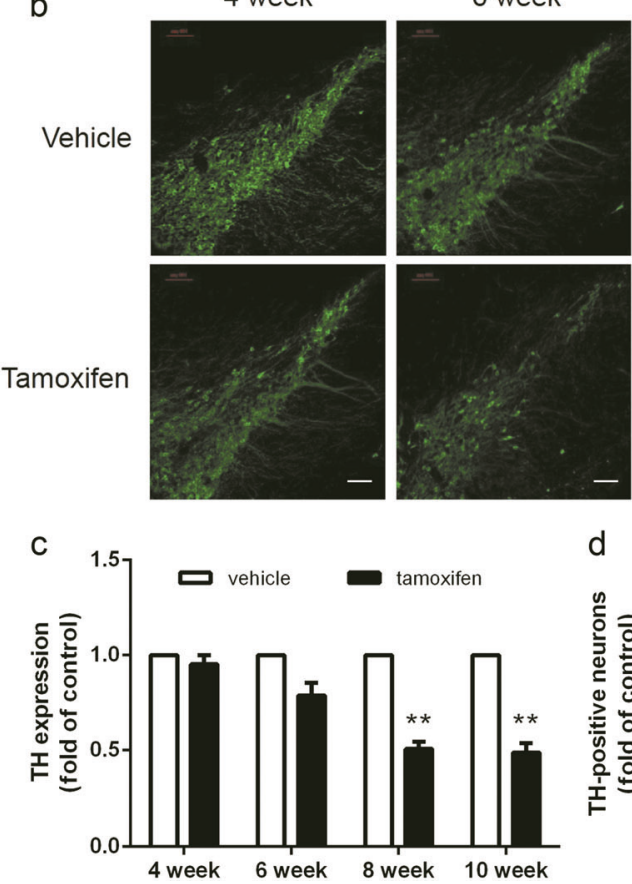

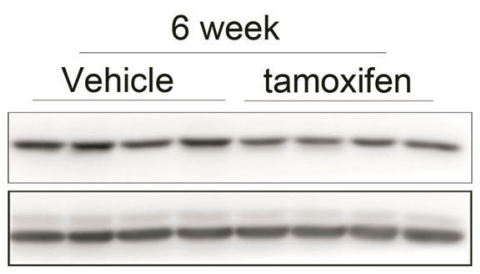

10 week

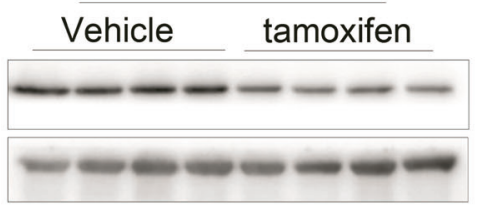

8 week
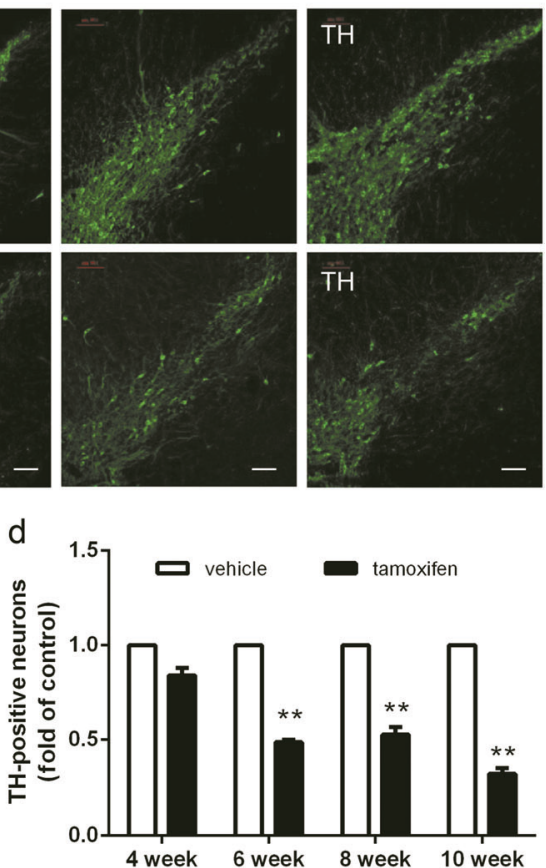

Fig. 2 Dicer CKO mice manifested a progressive reduction on TH expression in SN. Mice were sacrificed at indicated time points after tamoxifen administration. Mice were subjected to tissue preparations for either dissecting for western assay or perfusion for immunostaining, respectively as described in Methods. a The expression of TH in SN were measured by western blot. b The immunofluorescence staining with TH in SN. Representative photomicrographs were shown at $\times 10$ magnification (scale bars, $100 \mu \mathrm{m}$ ). c Quantitative analyses of TH expression and $\mathbf{d}$ counts of TH-positive neurons. Data were presented as mean \pm SEM. Statistical analyses were performed by two-way ANOVA. $n=4$ per group $(* * P<0.01 ;$ tamoxifen vs vehicle)

suppressed reactive microglia proliferation, as evidenced by the decreased number of Iba1-positive cells (Fig. 7c-e). Taken together, these results suggest that chronic DHM or PRE-084 treatment can protect DA neurons and reduce neuroinflammation in response to Dicer cKO.

\section{DISCUSSION}

In this study, we established an inducible DA neuron-specific Dicer cKO mouse line by crossing floxed Dicer mice (Dicer ${ }^{\mathrm{f} / \mathrm{f}}$ ) with DATicreER mice to obtain DAT-icreER;Dicer ${ }^{\mathrm{f} /+}$ mice, the latter were bred with Dicer ${ }^{\mathrm{f} / \mathrm{f}}$ mice to produce DAT-icreER;Dicer ${ }^{\mathrm{f} / \mathrm{f}}$ mice, which we called Dicer cKO mice. Following tamoxifen induction, DA neurons experienced progressive death, and a significant loss of DA neurons was observed 6 weeks after the depletion of Dicer in the SN. Accordingly, the behavioral tests revealed progressive
PD-like impairment of motor functions. In addition, we also observed time-dependent inflammation in the SN during the development of PD-like phenotypes, and microglia-mediated neuroinflammation was regarded as the hallmark of PD pathogenesis. These data revealed that Dicer CKO mice develop PD-like pathological and behavioral phenotypes, specifically in a progressive manner. The acute administration of $L$-DOPA to Dicer CKO mice significantly relieved the impairment of motor functions, indicating the potential of the Dicer CKO mouse model in evaluating PD drug efficacy. We further found that the chronic administration of DHM or a selective sigma-1 receptor agonist significantly attenuated the development of PD-like pathological and behavioral changes in Dicer CKO mice. Although the mechanisms of the respective drugs are different, this model can also be used to investigate the pathological mechanism of PD and to validate the effects of various antiparkinsonian drugs. 
a
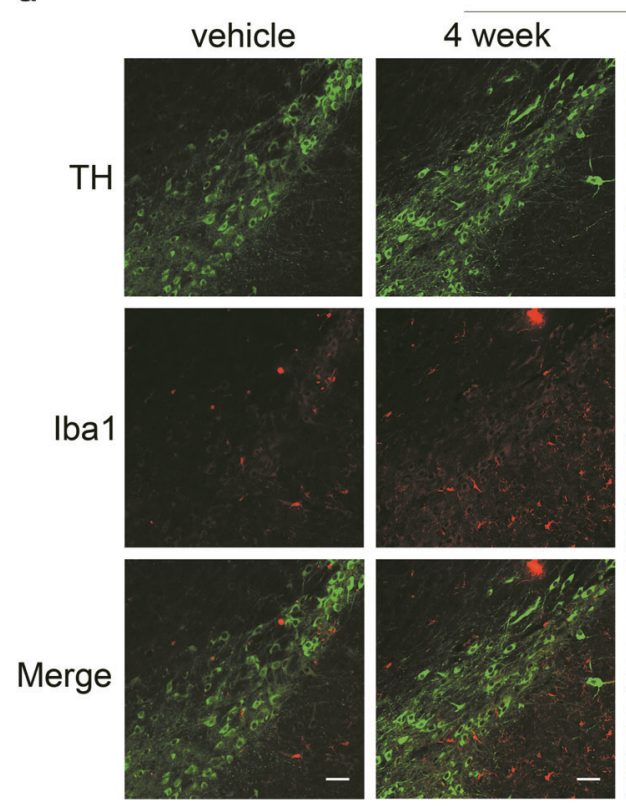

b
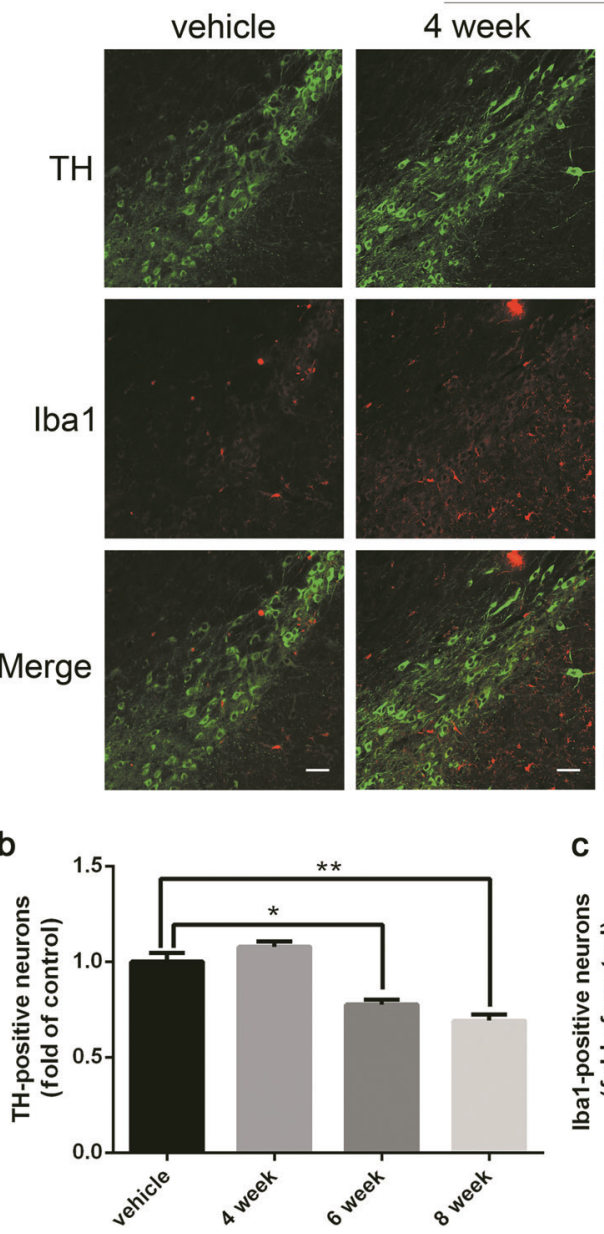

tamoxifen
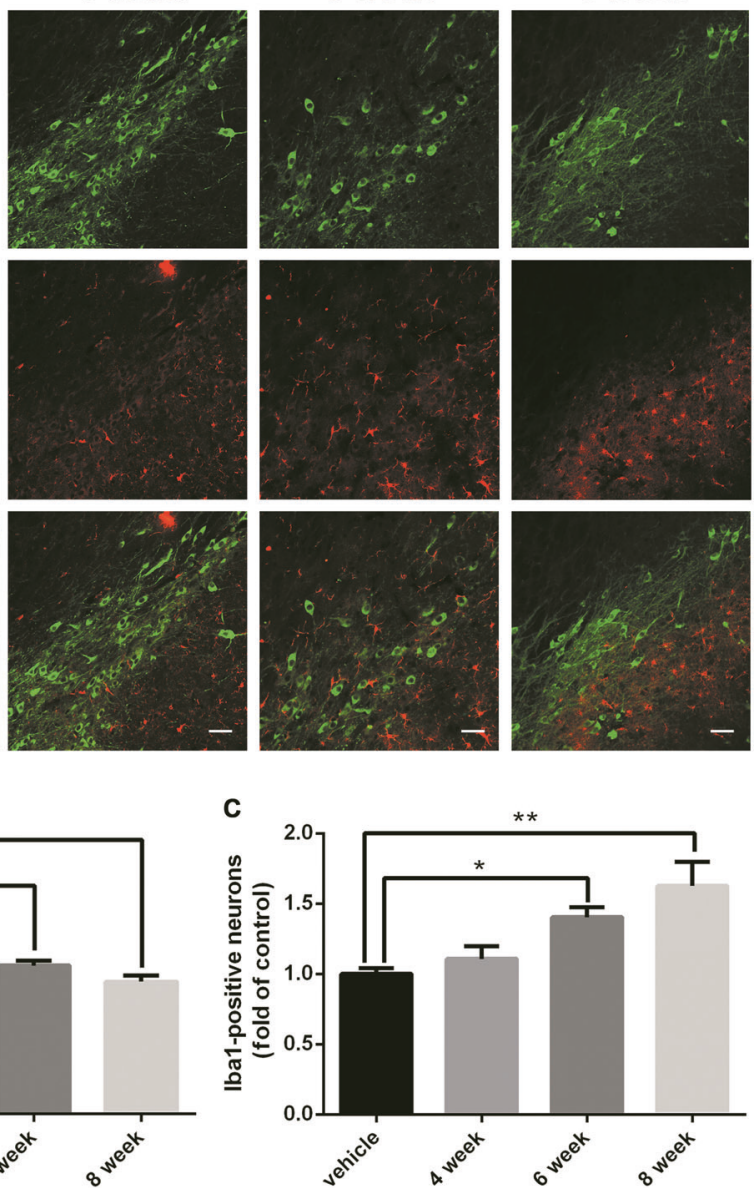

Fig. 3 Progressive neuroinflammation development in Dicer CKO mice. Animals were administrated with tamoxifen to induce the deletion of Dicer in DA neurons as depicted in Fig. 1. a Double immunofluorescence staining with respective anti-TH and anti-Iba1 antibodies were performed in brain sections at various time points. Iba1-positive-cell numbers in SN were increased with time in response to the Dicer cKO. Representative photomicrographs were shown at $\times 20$ magnification (scale bars, $100 \mu \mathrm{m}$ ). $\mathbf{b}$ Quantitative analyses of TH-positive neurons and c lbal-positive neurons. Data were presented as mean \pm SEM. Statistical analyses were performed by one-way ANOVA. $n=3$ per group ( ${ }^{*} P<$ $0.05,{ }^{*} P<0.01 ;$ tamoxifen vs vehicle)
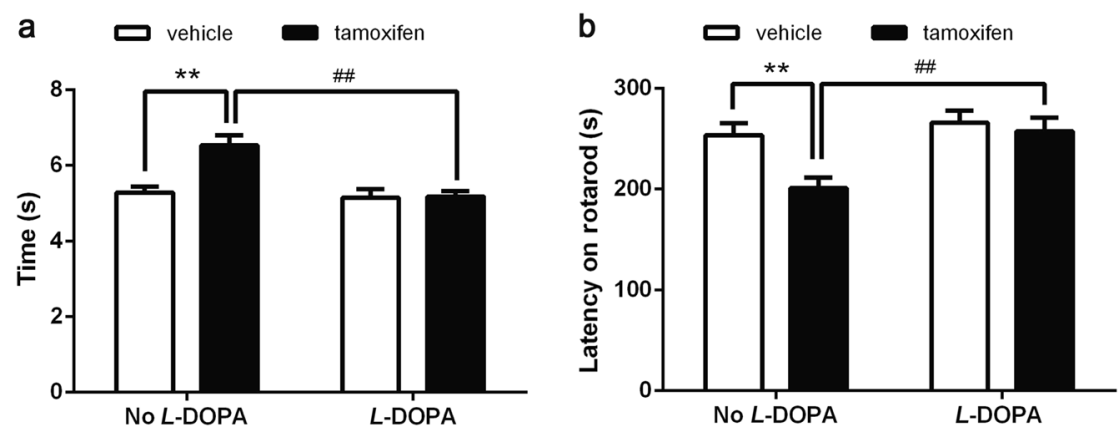

Fig. 4 L-DOPA alleviated the motor disorder in Dicer CKO mice. Eight weeks after tamoxifen or vehicle administration, mice were subjected to behavioral tests to monitor the motor impairments. Mice were then injected with $8 \mathrm{mg} / \mathrm{kg} L$-DOPA with $15 \mathrm{mg} / \mathrm{kg}$ benserazide before the behavioral tests. a Pole test and $\mathbf{b}$ Rotarod were tested $1 \mathrm{~h}$ later. Data were presented as mean \pm SEM. Statistical analyses were performed by two-way ANOVA. $n=10$ per group $\left({ }^{* *} P<0.01\right.$; No L-DOPA injection, vehicle vs tamoxifen. ${ }^{\# \#} P<0.01$; Dicer CKO mice, no L-DOPA vs L-DOPA)

miRNAs are single-stranded noncoding RNAs that bind to targeted gene sequences to inhibit target gene translation. Recently, many studies have revealed the important functional roles of miRNAs in neuronal differentiation, development, and function [18, 19]. Moreover, alterations in miRNAs have been widely reported to be associated with neuropsychiatric diseases $[20,21]$. For example, we recently reported that Let-7c and miRNA$3473 \mathrm{~b}$ are involved in microglial activation and contribute to neuronal survival and functional recovery in stroke [22, 23], indicating the importance of miRNAs in neuroprotection and 

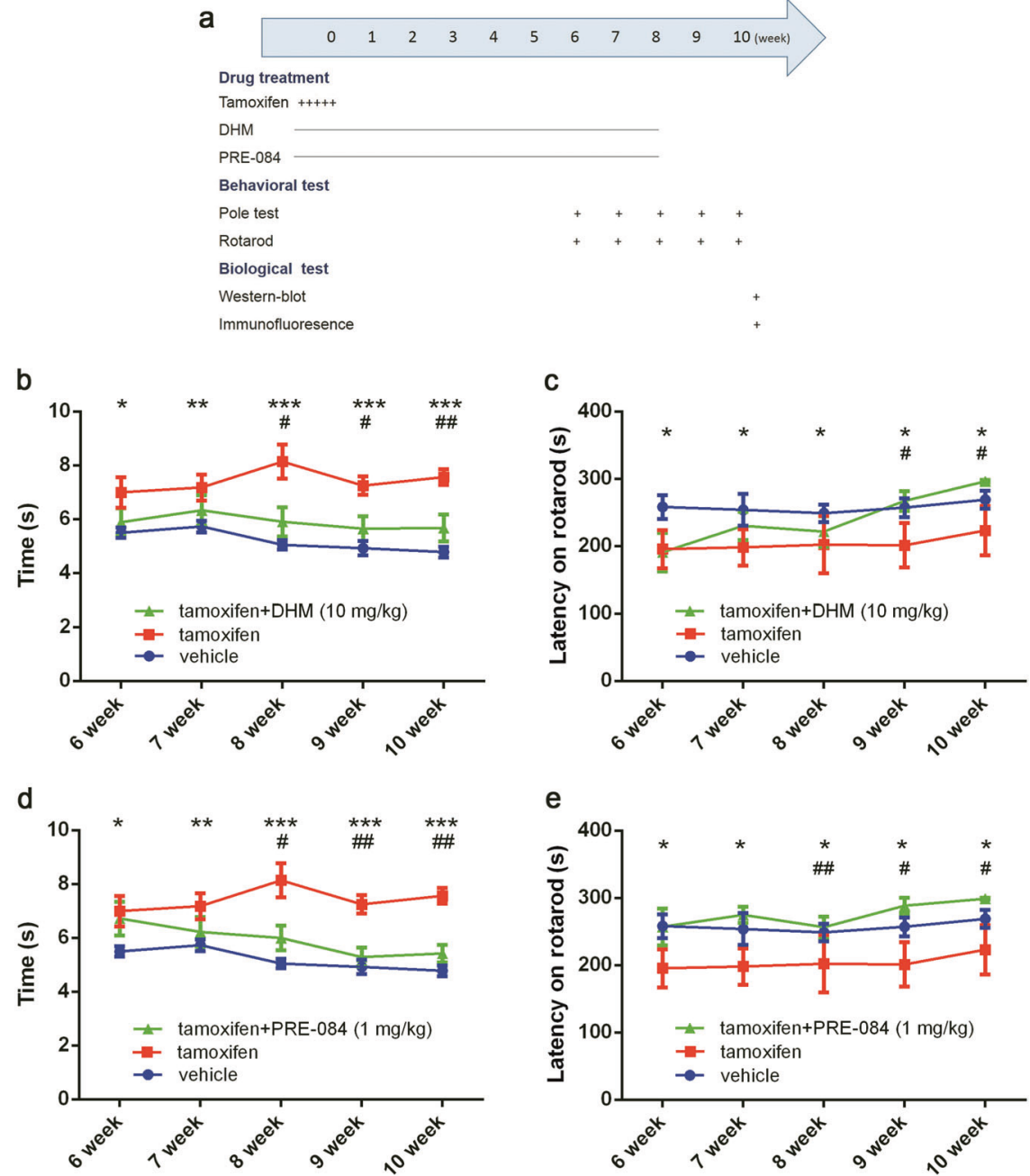

Fig. 5 Chronic DHM and PRE-084 treatment attenuated the development of PD-like motor functions in Dicer cKO mice. All mice were injected with $10 \mathrm{mg} / \mathrm{ml}$ tamoxifen (i.p.) twice a day for 5 days as described before. Meanwhile, mice also received daily injection $10 \mathrm{mg} / \mathrm{kg}$ of DHM, $1 \mathrm{mg} / \mathrm{kg}$ of PRE-084 or vehicle, respective for 8 weeks. The behavioral test were conducted every other week started from week 6 as described in Methods. a Flowchart of experimental procedures. b-e pole test and rotarod test were performed in mice at indicated times with or without DHM and PRE-084 injection, respectively. Data were presented as mean \pm SEM. Statistical analyses were performed by two-way ANOVA. $n=6$ per group $\left({ }^{*} P<0.05,{ }^{* *} P<0.01,{ }^{* *} P<0.001\right.$; tamoxifen vs vehicle. ${ }^{\#} P<0.05,{ }^{\# \#} P<0.01$; drugs vs tamoxifen)

neuroinflammation. In relation to the current study, alterations in miRNAs in PD pathological development have been widely studied, and circulating miRNAs have also been suggested to be potential novel biomarkers for PD. Moreover, it has been suggested that targeting specific miRNAs could be a potential therapeutic approach for PD. For instance, treatment with miR-124 agomir was shown to reduce the loss of DA neurons in an MPTPtreated mouse model, inhibiting Bim expression and thus suppressing Bax translocation to mitochondria [24]. Dicer is a type III RNase and is responsible for the processing of miRNA precursors into functional miRNAs, thus playing a crucial role in miRNA regulation [25]. Dicer expression is essential not only for midbrain development in mice and DA neuron maintenance and survival during early postnatal development, but also for DA neuron survival in adult mice [11]. Recently, a reduction in Dicer in the ventral midbrain and alterations in miRNA expression profiles in laser-microdissected DA neurons of aged mice have been demonstrated [26]. Moreover, miR-133b was reported to be specifically deficient in midbrain DA neurons from PD patient samples. It was further found that miR-133b alters the survival of DA neurons through negative feedback regulation with pitx3 [10].
Additionally, it was shown that the stimulation of miRNA biosynthesis promotes the survival of cultured DA neurons and reduces their vulnerability to thapsigargin-induced endoplasmic reticulum stress [26]. In agreement with these observations, our adult Dicer CKO mice show progressive loss of DA neurons in the $\mathrm{SN}$ in response to tamoxifen induction and consequently the presence of PD-like impairments in motor functions. We have characterized the detailed time-dependent changes and degeneration of DA neurons in the SN in response to the specific deletion of Dicer driven by DAT-Cre. A slightly different time course of PD-like phenotypes than that reported by a previous study that employed a different approach to delete Dicer from DA neurons, in which $90 \%$ DA loss was observed at week 8 . We found dramatic DA loss at week 10 following tamoxifen induction [10]. The specific mechanism by which Dicer cKO induces DA neuron loss remains to be further studied. In addition to the direct effect of Dicer depletion on DA neurons, we found that the number of lba1-positive cells in the SN increased significantly 6 weeks after tamoxifen injection, which was consistent with the time at which neurons began to die. The depletion of Dicer induced the activation of microglia-mediated neuroinflammation. Given that 
a

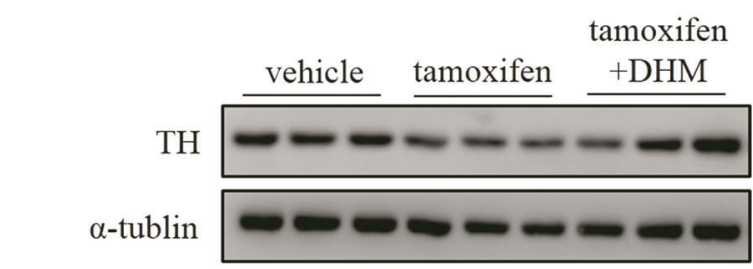

c
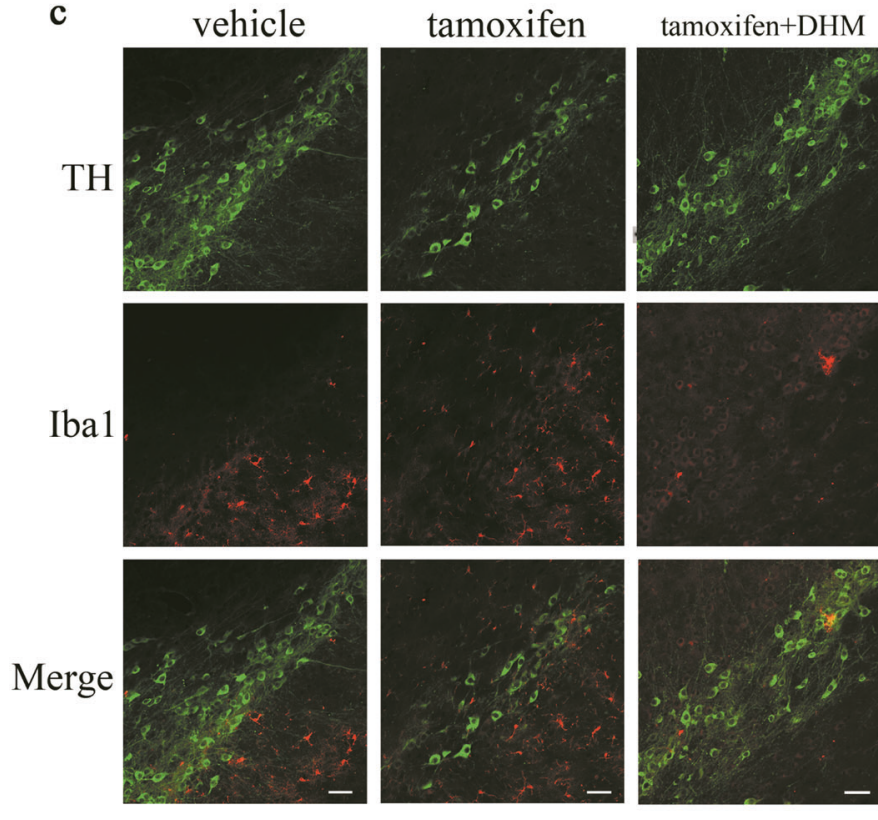

b

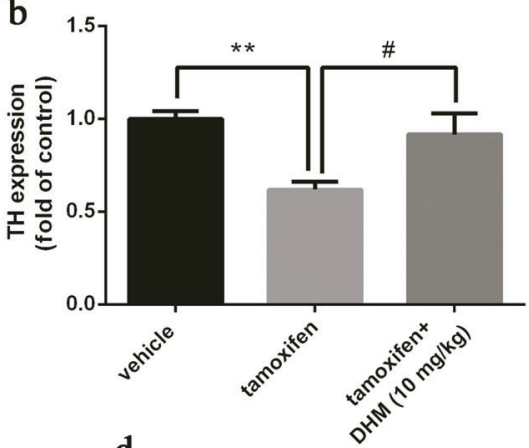

d
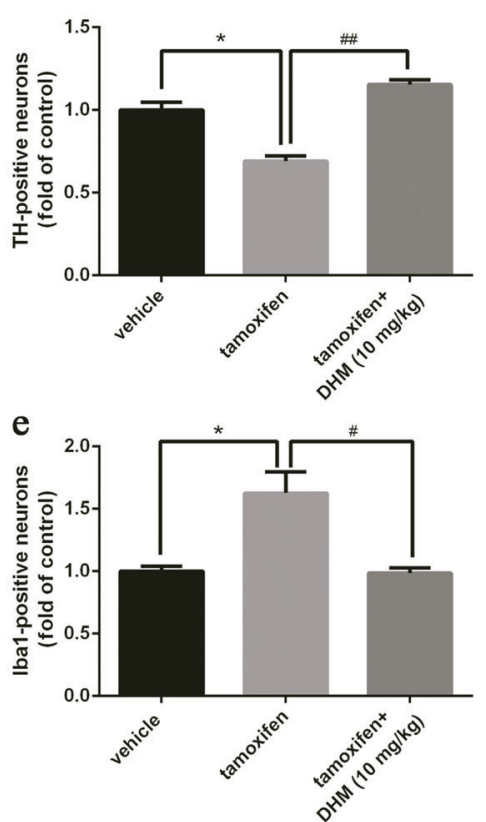

Fig. 6 Chronic DHM treatment alleviated DA neuron loss in Dicer cKO mice. After last behavioral tests as described in Fig. 5a, mice were sacrificed either for brain tissue dissection or for immunostaining as described in Methods. a, b TH expression was detected by anti-TH antibody using Western blot. c Immunostaining was conducted with respective antibody of TH and lba1. Representative photomicrographs were shown at $\times 20$ magnification (scale bars, $100 \mu \mathrm{m}$ ). d Quantitative analyses of TH-positive neurons and e lbal-positive neurons. Data were presented as mean \pm SEM. Statistical analyses were performed by one-way ANOVA. $n=3$ per group $\left({ }^{*} P<0.05,{ }^{*} P<0.01\right.$; tamoxifen vs vehicle. ${ }^{\#} P<0.05,{ }^{\# \#} P<0.01 ;$ DHM vs tamoxifen)

neuroinflammation is another hallmark of PD pathology and that miRNAs have been widely reported to be involved in the regulation of neuroinflammation [21], it is conceivable that Dicer depletion-mediated microglial activation may also contribute to DA neuron death.

In addition to pathological development, the mice also exhibited progressive impairments in motor functions that resembled the PD-like phenotypes. It was also noted that we did not detect any difference between males and females. Therefore, Dicer cKO mice may be an alternative PD animal model. Indeed, the acute application of L-DOPA significantly relieved motor impairments in response to Dicer cKO 8 weeks after tamoxifen induction. Furthermore, the chronic administration of neuroprotective agent DHM and the selective sigma- 1 receptor agonist PRE-084 significantly attenuated the development of PD-like behaviors and pathological phenotypes. All data indicate that Dice cKO mice may serve as a useful PD model.

The chronic application of the neuroprotective agent DHM has been shown to produce powerful neuroprotective effects in models of different diseases, including PD and stroke [13, 27]. As a traditional Chinese medicine, it was initially used to treat cough and fever and later found to have many pharmacological effects, such as protective effects on the heart, diabetes, liver, and nervous system, possibly through reducing oxidative stress or antiinflammation $[28,29]$. The neuroprotective effects of DHM were further suggested to be associated with its modulation of the AKTGSK3 $\beta$ signaling pathway [13]. In this study, we confirmed that DHM administration for 8 weeks reduced motor dysfunction, attenuated DA neuron death, and reduced microglial activation in mice DA neurons with Dicer cKO. The Sigma-1 receptor is an important target for a few CNS diseases [30, 31]. The sigma-1 receptor agonist PRE-084 can alleviate high spontaneous activity in mice and reduce DA outflow and release induced by acute methamphetamine administration [32]. In a chronic unpredicted mild stress model (CUMS), SOMCL-668, a recently discovered sigma-1 allosteric modulator, was reported to elicit an antidepressant effect through the BDNF-GSK3 pathway [31]. In addition, the administration of the low-dose sigma-1 receptor selective agonist PRE-084 for 5 consecutive weeks produced a good therapeutic effect in a 6-OHDA-induced PD model [17]. In agreement with these observations, we also found that 
a

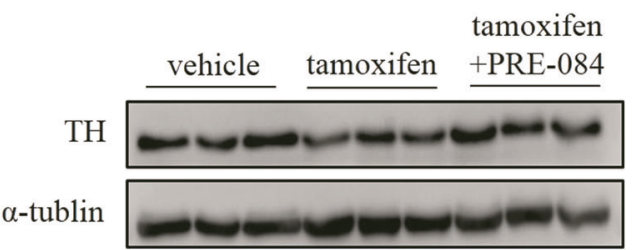

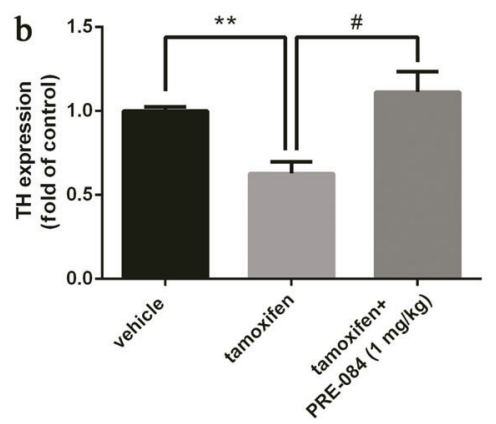

c vehicle
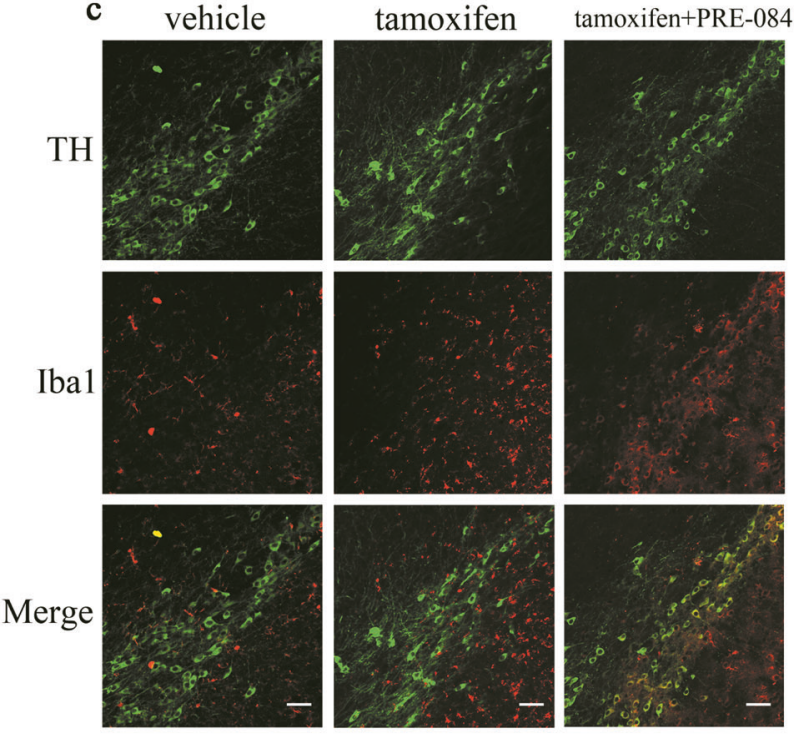

d
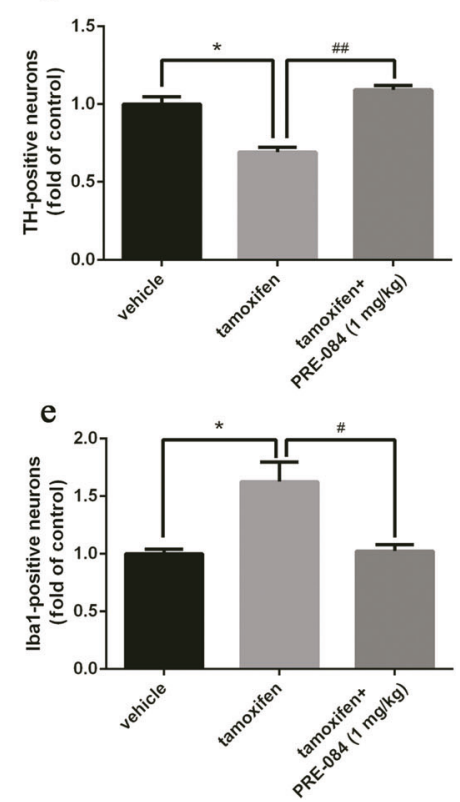

Fig. 7 Chronic PRE-084 treatment alleviated DA neuron loss in Dicer cKO mice. The experiments were conducted as described in Fig. 5a. a, b TH expression in SN. c TH and Iba1 immunostaining. Representative photomicrographs were shown at $\times 20$ magnification (scale bars, $100 \mu \mathrm{m}$ ). d Quantitative analyses of TH-positive neurons and e Ibal-positive neurons. Data were presented as mean \pm SEM. Statistical analyses were performed by one-way ANOVA. $n=3$ per group $\left({ }^{*} P<0.05,{ }^{* *} P<0.01\right.$; tamoxifen vs vehicle. ${ }^{\#} P<0.05$, ${ }^{\#} P<0.01 ;$ PRE-084 vs tamoxifen)

long-term administration of PRE-084 improved motor ability, protected DA neurons, and suppressed neuroinflammation, further confirming that our transgenic mouse model is valuable for evaluating the efficacy of PD drugs.

In summary, the present study demonstrated that adult DATicre;Dicer $^{\mathrm{f} / \mathrm{f}}$ transgenic mice develop progressive DA neuron loss and PD-like behaviors accompanied by progressive neuroinflammation following tamoxifen induction. The acute application of L-DOPA can improve the impaired motor ability of Dicer CKO mice. Therefore, we believe that this model could be used to simulate the progressive and irreversible pathogenesis of PD and to study the pathogenesis of PD. In addition, we evaluated DHM and PRE084, two compounds that have been shown to elicit antiparkinsonian effects through different mechanisms, and found that the chronic application of either DHM or PRE-084 attenuated the development of PD-like behavioral and pathological phenotypes, further implying that the use of this model is an alternative approach for PD drug discovery.

\section{ACKNOWLEDGEMENTS}

This work was supported by grants from the National Natural Science Foundation of China (Nos. 81373382, 81973334, and 31970909) and the Priority Academic Program Development of the Jiangsu Higher Education Institutes (PAPD). Support from the National Center for International Research (2017B01012) is also appreciated.

\section{AUTHOR CONTRIBUTIONS}

CHG, XCZ, and JLW designed the research and wrote the paper; CHG and TC performed the experiments; CHG and LTZ analyzed the data.

\section{ADDITIONAL INFORMATION}

Competing interests: The authors declare no competing interests.

\section{REFERENCES}

1. Radhakrishnan DM, Goyal V. Parkinson's disease: a review. Neurol India. 2018;66: S26-35.

2. Kalia LV, Lang AE. Parkinson's disease. Lancet. 2015;386:896-912.

3. Jackson-Lewis V, Przedborski S. Protocol for the MPTP mouse model of Parkinson's disease. Nat Protoc. 2007;2:141-51.

4. Becker B, Demirbas M, Johann S, Zendedel A, Beyer C, Clusmann H, et al. Effect of intrastriatal 6-OHDA lesions on extrastriatal brain structures in the mouse. Mol Neurobiol. 2018;55:4240-52.

5. Bartel DP. MicroRNAs: genomics, biogenesis, mechanism, and function. Cell. 2004;116:281-97.

6. Im HI, Kenny PJ. MicroRNAs in neuronal function and dysfunction. Trends Neurosci. 2012;35:325-34.

7. Hebert SS, Papadopoulou AS, Smith P, Galas MC, Planel E, Silahtaroglu AN, et al. Genetic ablation of Dicer in adult forebrain neurons results in abnormal tau hyperphosphorylation and neurodegeneration. Hum Mol Genet. 2010;19: 3959-69. 
8. Cheng S, Zhang C, Xu C, Wang L, Zou X, Chen G. Age-dependent neuron loss is associated with impaired adult neurogenesis in forebrain neuron-specific Dicer conditional knockout mice. Int J Biochem Cell Biol. 2014;57:186-96.

9. Schaefer A, O'Carroll D, Tan CL, Hillman D, Sugimori $M$, Llinas $R$, et al. Cerebellar neurodegeneration in the absence of microRNAs. J Exp Med. 2007;204: 1553-8.

10. Kim J, Inoue K, Ishii J, Vanti WB, Voronov SV, Murchison E, et al. A MicroRNA feedback circuit in midbrain dopamine neurons. Science. 2007;317:1220-4.

11. Pang X, Hogan EM, Casserly A, Gao G, Gardner PD, Tapper AR. Dicer expression is essential for adult midbrain dopaminergic neuron maintenance and survival. Mol Cell Neurosci. 2014;58:22-8.

12. Yan $Q$, Han $C$, Wang $G$, Waddington JL, Zheng $L$, Zhen $X$. Activation of AMPK/ mTORC1-mediated autophagy by metformin reverses Clk1 deficiency-sensitized dopaminergic neuronal death. Mol Pharmacol. 2017;92:640-52.

13. Ren ZX, Zhao YF, Cao T, Zhen XC. Dihydromyricetin protects neurons in an MPTPinduced model of Parkinson's disease by suppressing glycogen synthase kinase-3 beta activity. Acta Pharmacol Sin. 2016;37:1315-24.

14. Gu R, Zhang F, Chen G, Han C, Liu J, Ren Z, et al. Clk1 deficiency promotes neuroinflammation and subsequent dopaminergic cell death through regulation of microglial metabolic reprogramming. Brain Behav Immun. 2017;60:206-19.

15. Colonna M, Butovsky O. Microglia function in the central nervous system during health and neurodegeneration. Annu Rev Immunol. 2017;35:441-68.

16. Wang Q, Liu Y, Zhou J. Neuroinflammation in Parkinson's disease and its potential as therapeutic target. Transl Neurodegener. 2015;4:19.

17. Francardo V, Bez F, Wieloch T, Nissbrandt H, Ruscher K, Cenci MA. Pharmacological stimulation of sigma-1 receptors has neurorestorative effects in experimental parkinsonism. Brain. 2014;137:1998-2014.

18. Mouradian MM. MicroRNAs in Parkinson's disease. Neurobiol Dis. 2012;46: 279-84.

19. Davis TH, Cuellar TL, Koch SM, Barker AJ, Harfe BD, McManus MT, et al. Conditional loss of Dicer disrupts cellular and tissue morphogenesis in the cortex and hippocampus. J Neurosci. 2008;28:4322-30.

20. Cao T, Zhen XC. Dysregulation of miRNA and its potential therapeutic application in schizophrenia. CNS Neurosci Ther. 2018;24:586-97.
21. Varol D, Mildner A, Blank T, Shemer A, Barashi N, Yona S, et al. Dicer deficiency differentially impacts microglia of the developing and adult brain. Immunity. 2017;46:1030-44 e8.

22. Ni J, Wang $X$, Chen S, Liu H, Wang $Y, X u X$, et al. MicroRNA let-7c-5p protects against cerebral ischemia injury via mechanisms involving the inhibition of microglia activation. Brain Behav Immun. 2015;49:75-85.

23. Wang $X$, Chen S, Ni J, Cheng J, Jia J, Zhen X. miRNA-3473b contributes to neuroinflammation following cerebral ischemia. Cell Death Dis. 2018;9:11.

24. Wang H, Ye Y, Zhu Z, Mo L, Lin C, Wang Q, et al. MiR-124 regulates apoptosis and autophagy process in MPTP model of Parkinson's disease by targeting to Bim. Brain Pathol. 2016;26:167-76.

25. Wang Q, He Q, Chen Y, Shao W, Yuan C, Wang Y. JNK-mediated microglial DICER degradation potentiates inflammatory responses to induce dopaminergic neuron loss. J Neuroinflammation. 2018;15:184.

26. Chmielarz P, Konovalova J, Najam SS, Alter $\mathrm{H}$, Piepponen TP, Erfle H, et al. Dicer and microRNAs protect adult dopamine neurons. Cell Death Dis. 2017;8:e2813.

27. Zhao YF, Wang PP, Chen SS, Han CJ, Yan QT, Zheng LT, et al. Dihydromyricetin protects against cerebral ischemia/reperfusion injury via suppressing microgliamediated neuroinflammation and activation of ERK1/2-CREB signaling pathway. J Funct Foods. 2017;33:76-84.

28. Zhang J, Chen $Y$, Luo $H$, Sun $L, X u M, Y u ~ J$, et al. Recent update on the pharmacological effects and mechanisms of dihydromyricetin. Front Pharmacol. 2018;9:1204.

29. Liu TT, Zeng Y, Tang K, Chen X, Zhang W, Xu XL. Dihydromyricetin ameliorates atherosclerosis in LDL receptor deficient mice. Atherosclerosis. 2017;262:39-50.

30. Hong J, Sha S, Zhou L, Wang C, Yin J, Chen L. Sigma-1 receptor deficiency reduces MPTP-induced parkinsonism and death of dopaminergic neurons. Cell Death Dis. 2015;6:e1832.

31. Wang Y, Guo L, Jiang HF, Zheng LT, Zhang A, Zhen XC. Allosteric modulation of Sigma-1 receptors elicits rapid antidepressant activity. CNS Neurosci Ther 2016;22:368-77.

32. Sambo DO, Lin M, Owens A, Lebowitz JJ, Richardson B, Jagnarine DA, et al. The sigma-1 receptor modulates methamphetamine dysregulation of dopamine neurotransmission. Nat Commun. 2017;8:2228. 